\title{
Principais impactos psicológicos em crianças vítimas de abuso sexual
}

\author{
Main psychological impacts on children victims of sexual abuse \\ Principales impactos psicológicos en los niños víctimas de abuso sexual
}

Recebido: 16/06/2021 | Revisado: 24/06/2021 | Aceito: 25/06/2021 | Publicado: 10/07/2021

\author{
Pauliana Andrade Sousa \\ ORCID: https://orcid.org/0000-0003-1693-605X \\ Faculdade de Ensino Superior do Piauí, Brasil \\ E-mail: paulianadesousa8@gmail.com \\ Lígia Damasceno Cronemberger Neiva \\ ORCID: https://orcid.org/0000-0001-6502-3674 \\ Faculdade de Ensino Superior do Piauí, Brasil \\ E-mail: ligia.neiva1@gmail.com \\ Ruth Raquel Soares de Farias \\ ORCID: https://orcid.org/0000-0002-0988-0900 \\ Universidade Federal do Piauí, Brasil \\ E-mail: ruthraquelsf@gmail.com
}

\begin{abstract}
Resumo
O presente artigo buscou verificar os reflexos do abuso sexual na criança e os impactos psicológicos deixados em curto e a longo prazos. Assim, foi realizada uma discussão sobre as contribuições do acompanhamento e da intervenção do psicólogo no manejo a criança abusada. O procedimento metodológico contou com a realização de pesquisas bibliográficas de forma sistemática em materiais de referência na área, incluindo livros, artigos e referenciais legislativos nas bases de dados científicas Scientific Electronic Library Online (SciELO) e Literatura Científica e Técnica da América Latina e Caribe (LILACS). Por conseguinte, foi feita a correlação dos artigos e construído um panorama sobre a temática em questão no cenário pandêmico. Desta maneira, foi possível constatar através da literatura investigada que o abuso sexual é um dos tipos de violência que mais ocorre no ambiente familiar e talvez uma das mais difíceis de ser tratada, considerando que o dano físico e a integridade psicológica são consequências comuns.
\end{abstract}

Palavras-chave: Criança; Delitos sexuais; Trauma psicológico.

\begin{abstract}
This article sought to verify the effects of sexual abuse on children and the psychological impacts left in the short and long term. Thus, a discussion was held about the contributions of monitoring and the psychologist's intervention in handling abused children. The methodological procedure included systematic bibliographic research on reference materials in the area, including books, articles and legislative references. Therefore, the articles were correlated and an overview of the topic in question in the pandemic scenario was built.
\end{abstract}

Keywords: Kid; Sexual offenses; Psychological trauma.

\section{Resumen}

Este artículo buscó verificar los efectos del abuso sexual en la niñez y los impactos psicológicos dejados a corto y largo plazo. Así, se realizó una discusión sobre los aportes del seguimiento y la intervención del psicólogo en el manejo de los niños maltratados. El procedimiento metodológico incluyó una investigación bibliográfica sistemática sobre materiales de referencia en el área, incluyendo libros, artículos y referencias legislativas. Por lo tanto, los artículos se correlacionaron y se construyó una visión general del tema en cuestión en el escenario de la pandemia.

Palabras clave: Niño; Delitos sexuales; Trauma psicólogico.

\section{Introdução}

A partir da década de 1980 começou a surgir no Brasil preocupações sobre os cuidados e a violência acometidas com crianças, sendo considerada como um grave problema médico social. Na mesma década apareceram os primeiros diagnósticos de maus tratos com propostas de intervenção que levaria a criação do Estatuto da Criança e do Adolescente (ECA), decretado em julho de 1990. Após a efetivação do ECA foram realizadas várias pesquisas voltadas para a temática da violência e 
principalmente do abuso sexual contra crianças na tentativa de entender como ocorre e quais consequências desencadeadas por tais atos (Viodres; Ristum, 2008).

De acordo com o ministério da mulher, da família e dos direitos humanos a cada hora, três crianças são abusadas no Brasil, e cerca de $80 \%$ das vezes a violência acontece dentro de casa. Uma, em cada três a quatro meninas, será vítima de abuso e exploração sexual antes dos 18 anos, entre os meninos um em cada seis a dez (Governo Federal, 2020).

Em março de 2020, o Brasil foi surpreendido por uma doença, um vírus da covid-19 onde acabou sendo reconhecido pela organização mundial da saúde como uma grave doença de proporções pandêmicas. Essa doença ainda desconhecida começou a se alastrar e contaminar milhares de pessoas que, sem possibilidade de tratamento farmacológico, fez com que a Organização mundial da saúde (OMS) tomasse algumas atitudes na tentativa de controlar a disseminação do vírus impondo afastamento social como forma de evitar o contagio entre as pessoas (Platt et al., 2020).

Como consequência, as atividades cotidianas de crianças foram proibidas restringindo sua interação social ao ambiente intrafamiliar. Com isso sem o contato social com outras pessoas, as crianças acabaram perdendo a interação com pessoas de apoio onde se pudesse revelar o abuso sofrido. Devido ao isolamento social estudos apontam que os números de abusos sexuais infantis intrafamiliar podem ter aumentado, já que a criança nem tem para onde ir ou encontrar com pessoas que possa relatar o abuso e como consequência o número de denúncias acaba diminuindo (Ibidem, 2020).

Nos últimos anos o abuso sexual infantil passa a ser foco de pesquisas e tem recebido especial atenção dos meios de comunicação como um todo principalmente após o início da pandemia. Hoje, em muitos países, existem programas em desenvolvimento para estudo, prevenção e tratamento do abuso sexual infantil tais como a Associação Brasileira Multiprofissional de Proteção à Infância e à Adolescência (ABRAPIA), o Programa de Atenção à Vítima de Abuso Sexual (PAVAS), Centro de Estudos e Atendimento Relativos ao Abuso Sexual (CEARAS), entre outros (Franca, 2003).

O presente artigo buscou verificar os reflexos do abuso sexual na criança e os impactos psicológicos deixados em curto e a longo prazos. Assim, foi realizada uma discussão sobre as contribuições do acompanhamento e da intervenção do psicólogo no manejo a criança abusada. O procedimento metodológico contou com a realização de pesquisas bibliográficas de forma sistemática em materiais de referência na área, incluindo livros, artigos e referenciais legislativos. Por conseguinte, foi feita a correlação dos artigos e construído um panorama sobre a temática em questão no cenário pandêmico. Desta maneira, foi possível constatar através da literatura investigada que o abuso sexual é um dos tipos de violência que mais ocorre no ambiente familiar e talvez uma das mais difíceis de ser tratada, considerando que o dano físico e a integridade psicológica são consequências comuns.

\section{Metodologia}

Para alcançar os objetivos da pesquisa foi utilizado o método bibliográfico sistemático. Este método possibilita uma investigação de materiais já elaborados e existentes sobre o tema abordado. Conforme Sampaio (2007) a abordagem sistemática disponibiliza um resumo das evidencias encontradas nas pesquisas e um apanhado das informações escolhidas, com a finalidade de reunir dados compara-los simultaneamente buscando evidenciar analise e resposta sobre determinado tema (Koller et al., 2014).

Nesta pesquisa foram utilizados apenas dados de pesquisa que abordam especificamente sobre o tema escolhido. Quanto as seleções dos artigos foram incluídas os que foram escritos em português e publicados entre os anos de 2011 - 2021. Como critério de exclusão foram descartados os artigos cujo tema não foi continente a pesquisa. Os resultados encontrados foram analisados e comparados a finalidade de discutir e formular considerações importantes para o tema abordado nesse trabalho. 
Para uma seleção adequada das fontes que foram utilizadas no presente trabalho houve uma pesquisa de artigos científicos e livros, além de consultas em sites destinados a pesquisa cientifica, sendo utilizado os descritores delitos sexuais/criança, delitos sexuais/consequências, delitos sexuais/atendimento, delitos sexuais/pandemia, nas bases de dados Biblioteca eletrônica Scientific Electronic Library Online (SciELO) e Literatura Científica e Técnica da América Latina e Caribe (LILACS).

A seleção foi realizada inicialmente com leituras de títulos e resumos de artigos, aplicando critérios de inclusão exclusão. Considerando como método a revisão sistemática e considerando os descritores já mencionados, foram encontrados cento e quatro (104) artigos nas bases de dos já mencionados acima (Fluxograma 1).

Fluxograma 1: Quantidade de artigos conforme a base de dados.

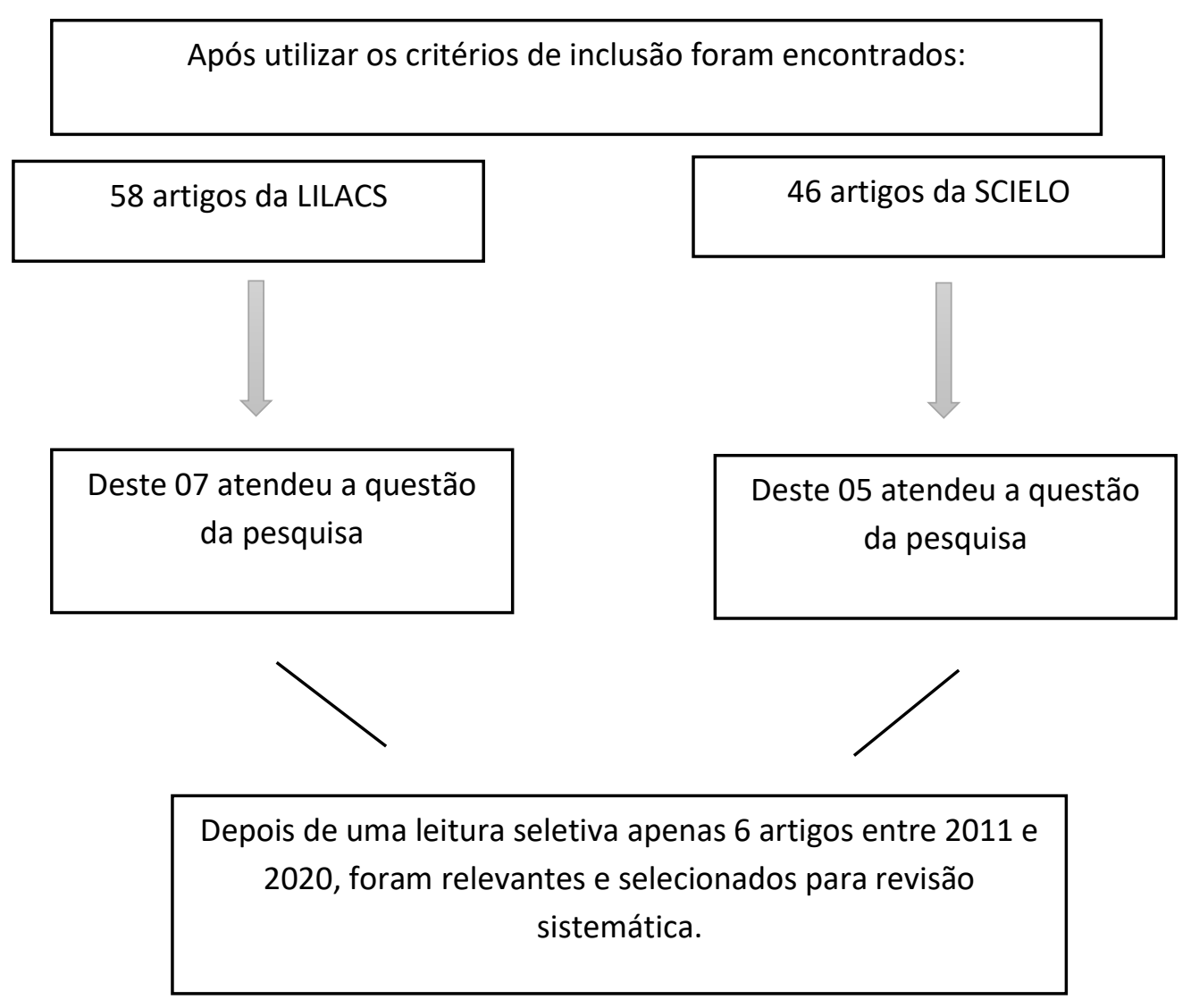

Fonte:Autores.

Posteriormente a seleção dos artigos utilizados na presente pesquisa houve uma necessidade da elaboração de um quadro no qual são inseridos autor e ano, o título, objetivo do artigo, metodologia e resultado/conclusão presentes nas fontes consultadas. 
Research, Society and Development, v. 10, n. 8, e18310817270, 2021

(CC BY 4.0) | ISSN 2525-3409 | DOI: http://dx.doi.org/10.33448/rsd-v10i8.17270

Quadro 1: Apresentação dos principais artigos selecionados.

\begin{tabular}{|c|c|c|c|c|}
\hline Autor/Ano & Título & Objetivo & Metodologia & Resultado ou conclusão \\
\hline $\begin{array}{l}\text { (Hohendorff; } \\
\text { Patias, 2017) }\end{array}$ & $\begin{array}{l}\text { Violência sexual contra } \\
\text { crianças e adolescente: } \\
\text { identificação, } \\
\text { consequências e } \\
\text { indicações de manejo }\end{array}$ & $\begin{array}{l}\text { O objetivo desse artigo é apresentar } \\
\text { informações essenciais sobre o fenômeno } \\
\text { da VS contra crianças e adolescentes aos } \\
\text { profissionais de áreas que costumam } \\
\text { intervir nesses casos (e.g., saúde, } \\
\text { assistência social, direito). }\end{array}$ & $\begin{array}{l}\text { Trata-se de uma metodologia } \\
\text { descritiva. }\end{array}$ & $\begin{array}{l}\text { Este artigo teve como objetivo oferecer informaçães sobre o } \\
\text { conceito de VS contra crianças e adolescentes, sobre a dinâmica } \\
\text { envolvida nessa forma de violência, sobre a legislação nacional } \\
\text { aplicada aos casos e sobre o manejo profissional diante da } \\
\text { revelação e dos casos de suspeita. Percebe-se que há uma } \\
\text { diversidade teórica na compreensão do que é VS contra crianças } \\
\text { e adolescentes e a dinâmica envolvida. No entanto, os autores } \\
\text { concordam que a VS deve ser conceituada de forma ampla, } \\
\text { considerando todos os atos que são infringidos às vítimas e dos } \\
\text { quais elas não são capazes de entender e dar seu consentimento. }\end{array}$ \\
\hline $\begin{array}{l}\text { (CRUZ et } \\
\text { al.,2019) }\end{array}$ & $\begin{array}{l}\text { Repercussões do abuso } \\
\text { sexual vivenciado na } \\
\text { infância e adolescência: } \\
\text { revisão integrativa }\end{array}$ & $\begin{array}{l}\text { O objetivo deste artigo é identificar as } \\
\text { repercussões do abuso sexual } \\
\text { experienciado na infância e na } \\
\text { adolescência. }\end{array}$ & $\begin{array}{l}\text { Trata-se de uma revisão integrativa de } \\
\text { literatura. }\end{array}$ & $\begin{array}{l}\text { Os estudos evidenciaram que pessoas que experienciam abuso } \\
\text { sexual na infância e na adolescência apresentam repercussões de } \\
\text { ordem psicológica, física, sexual e social, as quais perduram por } \\
\text { toda vida. O estudo oferece subsídios para alertar profissionais } \\
\text { de saúde quanto à necessidade de investigarem sinais e sintomas } \\
\text { sugestivos de abuso sexual, visto que experienciar tal agravo traz } \\
\text { inúmeras repercussões para vida das vítimas. }\end{array}$ \\
\hline $\begin{array}{l}\text { (AZNAR- } \\
\text { BLEFARI et al., } \\
\text { 2020) }\end{array}$ & $\begin{array}{l}\text { Atuação de Psicólogos } \\
\text { em Alegações de } \\
\text { Violência Sexual: Boas } \\
\text { Práticas nas Entrevistas } \\
\text { de Crianças e } \\
\text { Adolescentes }\end{array}$ & $\begin{array}{l}\text { O objetivo deste artigo é discutir a escuta } \\
\text { do psicólogo/a sobre alegações de } \\
\text { violência sexual nos contextos da escuta } \\
\text { especializada, do depoimento especial e } \\
\text { da perícia psicológica. O artigo também } \\
\text { tem como objetivo apresentar diretrizes } \\
\text { gerais para entrevistas com crianças e } \\
\text { adolescentes, consideradas na literatura } \\
\text { como boas práticas nesse campo de } \\
\text { atuação. }\end{array}$ & Trata-se de revisão narrativa. & $\begin{array}{l}\text { A qualificação de profissionais para a condução de entrevistas } \\
\text { com crianças nesses contextos é fundamental para que se garanta } \\
\text { a não revitimização, conforme previsto na recente legislação. No } \\
\text { entanto, ainda que careça de diretrizes específicas, as regras } \\
\text { básicas de entrevista com crianças e adolescentes também são } \\
\text { válidas no trabalho do profissional que atua com a escuta } \\
\text { especializada. Observou-se a necessidade de qualificação dos } \\
\text { psicólogos para a atuação nessas entrevistas, bem como a } \\
\text { necessidade da realização de estudos nesses diferentes contextos } \\
\text { de trabalho e procedimentos. }\end{array}$ \\
\hline $\begin{array}{l}\text { (LIRA et al., } \\
\text { 2017) }\end{array}$ & $\begin{array}{l}\text { Abuso sexual na } \\
\text { infância e suas } \\
\text { repercussões na vida } \\
\text { adulta }\end{array}$ & $\begin{array}{l}\text { O objetivo deste artigo é compreender } \\
\text { repercussões do abuso sexual na vida } \\
\text { adulta de mulheres abusadas sexualmente } \\
\text { na infância. }\end{array}$ & $\begin{array}{l}\text { Pesquisa qualitativa, desenvolvida com } \\
\text { nove mulheres em um Centro de } \\
\text { Referência da Mulher, na região do } \\
\text { semiárido do Estado de Pernambuco, } \\
\text { Brasil, através de entrevistas não } \\
\text { estruturadas. A interpretação dos } \\
\text { resultados foi fundamentada na } \\
\text { abordagem da Sociologia } \\
\text { Compreensiva e do Cotidiano, a fim de } \\
\text { identificar a centralidade subterrânea } \\
\text { submersa na vida cotidiana das }\end{array}$ & $\begin{array}{l}\text { Conclui-se que a exposição ao abuso sexual no contexto familiar } \\
\text { prejudicou a saúde física e emocional de meninas e adolescentes, } \\
\text { bem como a convivência familiar, apontando para a necessidade } \\
\text { de adoção de sensibilidade e solidariedade no cuidado a } \\
\text { mulheres com queixas que possam estar associadas a vivências } \\
\text { de abuso sexual. }\end{array}$ \\
\hline
\end{tabular}


Research, Society and Development, v. 10, n. 8, e18310817270, 2021

(CC BY 4.0) | ISSN 2525-3409 | DOI: http://dx.doi.org/10.33448/rsd-v10i8.17270

\begin{tabular}{|c|c|c|c|c|}
\hline & & & $\begin{array}{l}\text { participantes e que pudesse emergir por } \\
\text { meio de suas reações a partir do vivido } \\
\text { do abuso sexual. }\end{array}$ & \\
\hline $\begin{array}{l}\text { (MOREIRA; } \\
\text { MAGALHÃES, } \\
\text { 2020) }\end{array}$ & $\begin{array}{l}\text { Os impactos da } \\
\text { pandemia de covid-19 } \\
\text { no enfrentamento da } \\
\text { violência sexual } \\
\text { intrafamiliar contra } \\
\text { crianças e adolescentes }\end{array}$ & $\begin{array}{l}\text { O objetivo geral da pesquisa é analisar a } \\
\text { exploração sexual comercial de crianças e } \\
\text { adolescentes nas rodovias brasileiras. } \\
\text { Como objetivos específicos, estabeleceu- } \\
\text { se: conceituar a violência sexual } \\
\text { intrafamiliar contra crianças e } \\
\text { adolescentes; verificar suas causas e } \\
\text { agressores; apresentar o contexto da } \\
\text { acentuação da violência sexual } \\
\text { intrafamiliar na pandemia de Covid-19 e } \\
\text { seus impactos. }\end{array}$ & $\begin{array}{l}\text { O método de abordagem é o dedutivo e } \\
\text { o método de procedimento é o } \\
\text { monográfico. A técnica de pesquisa é a } \\
\text { bibliográfica, onde se teve como } \\
\text { alicerce o estudo de artigos científicos, } \\
\text { teses e livros. }\end{array}$ & $\begin{array}{l}\text { Constata-se, com o desenvolvimento do estudo, a confirmação } \\
\text { da hipótese estabelecida inicialmente, ou seja, o Brasil } \\
\text { consolidou a proteção jurídica para enfrentar a violência sexual } \\
\text { intrafamiliar contra crianças e adolescentes, no entanto são } \\
\text { muitos os desafios das políticas públicas intersetoriais em vista } \\
\text { das complexidades do problema, estando-se ainda mais } \\
\text { acentuadas em decorrência da pandemia de Covid-19. }\end{array}$ \\
\hline $\begin{array}{l}\text { (PLATT; } \\
\text { GUEDERT; } \\
\text { COELHO, 2020) }\end{array}$ & $\begin{array}{l}\text { Violência contra } \\
\text { crianças e adolescentes: } \\
\text { notificações e alerta em } \\
\text { tempos de pandemia }\end{array}$ & $\begin{array}{l}\text { O objetivo deste estudo visou avaliar e } \\
\text { comparar as notificações compulsórias de } \\
\text { violências interpessoais/autoprovocadas } \\
\text { disponíveis no Sistema de Informação de } \\
\text { Agravos de Notificação do Estado de } \\
\text { Santa Catarina, pré e pós-pandemia do } \\
\text { novo coronavírus. }\end{array}$ & $\begin{array}{l}\text { Estudo transversal, descritivo e } \\
\text { analítico das violências contra crianças } \\
\text { e adolescentes }\end{array}$ & $\begin{array}{l}\text { Alerta-se para a necessidade de a sociedade estar atenta para a } \\
\text { suspeita e evidência dos casos de violência na população } \\
\text { infantojuvenil, e ressalta-se a importância de que sejam } \\
\text { propiciadas formas acessíveis, eficazes e seguras, como } \\
\text { incentivo para as denúncias, a notificação e o rápido atendimento } \\
\text { dos casos, visando à proteção das vítimas, à minimização dos } \\
\text { danos e, assim, ao impedimento da perpetuação da violência. }\end{array}$ \\
\hline
\end{tabular}

Fonte:Autores. 


\section{Resultados e Discussão}

O respectivo estudo almejou, verificar os reflexos do abuso sexual no desenvolvimento psicológico da criança. Pare esse feito, foi realizada uma investigação de estudos sobre a temática em questão. De tal forma, a busca sucedeu na identificação de cento e quatro (104) artigos ao todo nas duas bases de busca (SciELO e LILACS). Contudo, como mencionado anteriormente, apenas seis atenderam aos critérios de seleção para a conferência e consequentemente para a análise teórica.

Nesse sentido, é possível inferir que todos os materiais revisados apresentaram consenso sobre os danos físicos e mentais que são desencadeados pelo abuso sexual. Da mesma maneira, os autores Ferreira e Nantes (2017), consideram o grande impacto ocasionado na vida da criança, que consequentemente podem se manifestar de diferentes formas e persistirem ao longo da vida.

Ainda sobre o que dizem os autores Ferreira e Nantes (2017), o abuso sexual é constituído de uma longa história, que por sua vez, é complexa, por envolver um cenário de dúvidas, incertezas, medos e problemas com os membros que envolve não somente com a vítima, mas também dos membros familiares. Por essa razão, se faz necessário e essencial um acompanhamento e uma intervenção psicológica tanto para vítima, como para seus familiares, para que possam oferecer um manejo adequado de suporte emocional, especialmente quando se trata de crianças.

Sobre os abusos com crianças, Platt et al., (2018), esclarece que a violência ocorre a partir do momento em que ela é submetida à atividade sexual a qual não possa entender, pois o nível em que se encontra o seu desenvolvimento é incompatível ao do abusador, impossibilitando assim o seu consentimento.

Ressalta-se que as consequências podem ser latentes e perceptíveis, mesmo no decorrer de tempos após o abuso, inclusive como resposta de uma crise evolutiva ou situacional e principalmente diante de estresse (Amazarray; Koller, 1998). As consequências do abuso sexual para crianças possuem mediadores do impacto, ou seja, a presença ou ausência desses mediadores vai interferir na apresentação e intensidade dos sintomas e psicopatologias.

Aznar-Blefari et al. (2020), reconhecem que a psicologia tem um importante papel na escuta qualificada de crianças e adolescentes vítimas de violência sexual. O que pode ser reafirmado com a pesquisa qualitativa de Lira et al. (2017), do projeto piloto intitulado "Oficinas interventivas com mulheres vítimas de violência doméstica”, em que vivenciaram a experiência de abuso sexual na infância praticada por um homem do seu convívio familiar.

Em face a essa realidade, os autores ibidem (2017) evidenciaram que um "imaginário de medo normalmente atravessado pelo silêncio, mulheres que foram abusadas sexualmente na infância guardam uma centralidade subterrânea, na qual se escondem alterações nocivas a sua vida cotidiana”.

Ressalta-se que diante do fechamento das escolas e de outros espaços importantes para a construção de vínculos de confiança com adultos fora de casa, crianças e adolescentes ficaram ainda mais vulneráveis à violência sexual durante a pandemia da Covid-19, como evidenciou o Fundo das Nações Unidas para a Infância (UNICEF), o Instituto Sou da Paz e o Ministério Público do Estado de São Paulo (MPSP). Esse foi um dos achados do estudo produzido pelas três organizações, que teve como objetivos verificar os possíveis impactos do isolamento social na ocorrência e na notificação da violência sexual e dar visibilidade a esse grave e recorrente problema e à necessidade de avançar no enfrentamento.

Portanto, de acordo com a literatura analisada e em consenso com a argumentação de todos os autores, é reafirmado nas palavras dos ilustres Hohendorf; e Patias (2017), que "as consequências da violência sexual contra crianças e adolescentes indicam a necessidade de atendimento às vítimas”. Assim, o papel dos profissionais que, de alguma forma, têm contato com a criança ou adolescente, é de extrema relevância (Ibidem, 2017). 


\section{Conclusão}

Diante do que foi exposto neste estudo, observa-se que o abuso sexual é um dos tipos de violência que ocorre no ambiente familiar e talvez uma das mais difíceis de ser tratada, considerando que o dano físico e a integridade psicológica são consequências comuns.

Em casos de revelação do abuso, é importante que a vítima se sinta acolhida e segura para falar sobre a violência vivenciada, bem como, receba crédito e apoio, pois se trata de um momento muito difícil.

É necessário ainda, que existam profissionais capacitados para identificar os casos de abuso sexual contra crianças e trabalhar tanto com intervenções preventivas, quanto terapêuticas, para a superação desta violência por parte da vítima e do grupo familiar como um todo.

Portanto, novos estudos sobre a temática são imprescindíveis para o desenvolvimento de mecanismos voltados à proteção integral de crianças e adolescentes. O enfrentamento perpassa um olhar integrado e multidisciplinar, envolvendo os atores da sociedade como um todo. Superar toda forma de violência contra crianças e adolescentes e, em particular, superar a violência sexual, por tudo já apresentado, não significa necessariamente extingui-la, mas consiste principalmente em criar mecanismos para promover a visibilidade aos direitos humanos e aos dispositivos de enfrentamento.

\section{Referências}

Amazarray, M. R. \& Koller, S. H. (1998). Alguns aspectos observados no desenvolvimento de crianças vítimas de abuso sexual. Psicologia: Reflexões e Critica. 11(3), 559-78.

Azevedo, M. A. N. \& Guerra, V. N. A. (1989). Crianças vitimizadas: a síndrome do pequeno poder. 2,ed. Ed. Iglu.

Azevedo, M.A.; Guerra, V. N. A. (1988). Pele de asno não é só história... Um estudo sobre a vitimização de crianças e adolescentes em família. Edição 1. São Paulo: Roca.

Aznar-blefari, C. et al. (2020). Atuação de Psicólogos em Alegações de Violência Sexual: Boas Práticas nas Entrevistas de Crianças e Adolescentes. PsicoUSF. 25(4), 625-35, Out.

Borges, Jeane Lessinger; Dell'aglio, Débora Dalbosco. (2008). Relações entre abuso sexual na infância, transtorno de estresse pós-traumático (TEPT) e prejuízos cognitivos. Psicol. estud. Maringá, v. 13, n. 2, p. 371-379, junho.

Brito, M. A. N. et al. (2005). Violência doméstica contra crianças e adolescentes: estudo de um programa de intervenção. Ciência saúde coletiva. Rio de Janeiro, v. 10, n.1, p.143-149, março.

Conselho federal de psicologia. (2009). Serviço de proteção social a criança e adolescentes vítimas de violência, abuso e exploração sexual e suas famílias: referencias para a atuação do Psicólogo, Brasília-DF.

Cogo, K., Et al. (2011). Consequências psicológicas do abuso infantil. Unoesc e Ciência, Joaçaba, v.2, n. 2, p. 130-139, jul.

Cruz, M. A., Gomes, N. P., Campos, LM, Estrela, F. M., Whitaker, M. C. O., Lírio, J. G. S. (2020). Repercussões do abuso sexual vivenciado na infância e adolescência: revisão integrativa. Revista Ciência Saúde Coletiva, abril.

Sociedade Brasileira de Pediatria. (2021). Combate ao Abuso e à Exploração Sexual e Outras Violências Contra Crianças e Adolescentes em Tempo da Quarentena por COVID-19. Departamento Científico de Segurança. Sociedade Brasileira de Pediatria, 2019-2021.

Day, V. P., et al. (2003). Violência doméstica e suas diferentes manifestações. Revista de psiquiatria do Rio Grande do Sul, Porto Alegre, v. 25, supl. 1, p. 921. Abril.

Ferreira, T.; Nantes, E. (2017). Contribuições da Psicologia Perante o Fenômeno de Abuso Sexual Infantil. Simpósio internacional em Educação Sexual, Campo Mourão-PR.

Florentino, B. R. B. (2015). As possíveis consequências do abuso sexual praticado contra crianças e adolescentes. Fractal, Rev. Psicol., Rio de Janeiro, v. 27, n. 2, p. 139-144. Agosto.

Franca junior, I. (2003). Abuso sexual na infância: compreensão a partir da Epidemiologia e dos Direitos Humanos. Interface (Botucatu). Botucatu, v. 7, n. 12, pág. 23-38, fevereiro.

Habigzang, L. F., et al. (2008). Avaliação psicológica em casos de abuso sexual na infância e adolescência. Revista Psicol. Reflexo. Crit., Porto Alegre, v. 21, n. 2, pág. 338-344.

Hohendorff, J, E; Patias, N, D. (2014). Violência sexual contra crianças e adolescentes: identificação, consequências e indicações de manejo. In: KOLLER, S. H.; DINIZ, E.; HABIGZANG, L. F. (Eds.) Trabalhando com adolescentes: Uma perspectiva bioecológica e aplicada (pp. 293-308). Porto Alegre: Artmed. 
Research, Society and Development, v. 10, n. 8, e18310817270, 2021

(CC BY 4.0) | ISSN 2525-3409 | DOI: http://dx.doi.org/10.33448/rsd-v10i8.17270

Lira, M. O. S. C. e et al. (2017). Abuso sexual na infância e suas repercussões na vida adulta. Texto contexto - Revista de Enfermagem, Florianópolis, v. 26, n. 3 .

Minayo, M. C. S. (1994). Violência social sob a perspectiva da saúde pública. Saúde Pública, Rio de Janeiro, v. 10, supl. 1,pág. S7-S18.

Ministério da Mulher, da Família e dos Direitos Humanos. (2020). Ministerio divulga dados de violência sexual contra crianças e adolescentes. Brasília, 18 de mai.

Moreira, R.; Magalhães, D. (2020). Os impactos da pandemia de covid-19 no enfrentamento da violência sexual intrafamiliar contra crianças e adolescentes. Revista da Jornada de Pós-graduação e Pesquisa. ISSN: 2526-4397 Congrega Urcamp, vol. 16, nº16.

Passarinho, M. С. B. T. (2015). O fenómeno do abuso sexual de crianças: O abusador intra-familiar e o extra-familiar. Tese de Doutorado.

Platt, V. B. et al. (2018). Violência sexual contra crianças: autores, vítimas e consequências. Ciênc. saúde coletiva, Rio de Janeiro, v. 23, n. 4, p. 1019-1031, Abril. https://doi.org/10.1590/1413_81232018234.11362016.

Pfeiffer, L.; Salvagni, E. P. (2005). Visão atual do abuso sexual na infância e adolescência. J. Pediatr. (Rio J.), Porto Alegre, v. 81, n. 5, supl. p. s197-s204, novembro.

Perty Froner, J.; Rohnelt, R.; Regina, V. (200). A escuta de crianças vítimas de abuso sexual intrafamiliar na concepção de profissionais que atuam no âmbito do Judiciário. Psicol. rev. (Belo Horizonte), Belo Horizonte, v.5, n.3, p.60-81, dez.

Sampaio, R. F., Mancini, M. C. (2007). Estudos de revisão sistemática: um guia para síntese criteriosa da evidência científica. Rev Bras Fisioter.

Sanderson, C. (2005). Abuso sexual em crianças: fortalecendo pais e professores para proteger crianças de abusos sexuais. São Paulo, M. Books.

Viodres, S.; Ristum, M. (2008). Violência sexual: caracterização e análise de casos revelados na escola. Estudos de Psicologia I .Campinas 25(1) I 11-21 I janeiro - março. 